\title{
Retrospective evaluation of clinical and pathological features, as well as diagnostic and treatment protocols of primary vaginal malignancy
}

\author{
Gozde Kulhan 1, 2, Mehmet Kulhan 1, 2, Umit Nayki², Cenk Nayki², Pasa Ulug' ${ }^{2}$, Emrah Akkaya', \\ Onur S. Aldemir ${ }^{1}$, Yusuf Yildirim ${ }^{1,2}$, Mehmet Sipahi ${ }^{1}$ \\ ${ }^{1}$ Tepecik Obstetrics and Gynecology Training and Research Hospital, Obstetrics and Gynecology Unit, Izmir, Turkey \\ ${ }^{2}$ Erzincan University Medical Faculty, Gynecology and Obstetrics Department, Erzincan, Turkey
}

\begin{abstract}
Objectives: Our study aimed to determine the epidemiological characteristics and diagnostic and treatment protocols of primary vaginal carcinoma. Also, we compared the clinical and pathological features of primary vaginal carcinoma which intersect with cervical and vulvar carcinomas.

Material and methods: Sixteen patients with primary vaginal carcinoma, admitted to the Department of Gynecologic Oncology, Izmir Tepecik Training and Research Hospital between January 1983 and December 2012, were evaluated retrospectively. FIGO (International Federation of Gynecology and Obstetrics) Surgical staging was performed. The SPSS (Statistical Package for Social Sciences) program was used for statistical analyses.

Results: The histological distribution of the 16 patients with primary vaginal carcinoma was as follows: 9 patients (56.3\%) with squamous cell carcinoma (SCC), 5 (31.3\%) with rhabdomyosarcoma, and 2 (12.5\%) with adenocarcinoma.

Conclusion: The stage of the disease was found the be the main factor affecting the survival.

Key words: primary vaginal malignancy, squamous cell carcinoma, sarcoma
\end{abstract}

Ginekologia Polska 2016; 87, 8: 541-545

\section{INTRODUCTION}

Primary vaginal cancer is a rare tumor of the female genital system as vaginal tissue is relatively resistant to malignant change. Primary vaginal cancer, first identified as a distinct type of cancer in 1952 by Graham and Meigs [1], accounts for approximately $1-2 \%$ of all gynecologic tumors [2]. The incidence of invasive vaginal cancer has been estimated at 0.42 per 100,000 women, and has remained stable since the 1980s [3]. Interestingly, malignancies, at both ends of the vaginal tract, are more often observed than vaginal malignant diseases. Metastatic cancers account for the vast majority (80-90\%) of vaginal malignancies [4]. Sixteen percent of vaginal cancers are primary, while $84 \%$ are metastatic. Twenty-seven percent of vaginal squamous cell carcinoma (SCC) cases are primary. However, $83 \%$ were found to be metastatic from the cervix or the vulva [5-7]. SCC accounts for the majority of the primary lesions. Mela- noma, sarcoma, and adenocarcinoma have been described in primary cancers as well. Sarcomas sometimes occur after radiotherapy for cervical cancer [8,9]. If cervical and vaginal involvement is detected together, it is assumed to be primary cervical carcinoma, whereas if vulvar and vaginal involvement is determined together, the cancer is assumed to be primary vulvar malignancy. Therefore, primary vaginal cancer is found in an extremely limited number of cases and is accompanied by the uncertainty of the primary diagnostic criteria. Two criteria are required in order for cancer to be accepted as primary, i.e. no pathology in the cervix or the vulva, and histopathological findings different from uterine tumors if hysterectomy was performed for uterine tumors within 5 years [10]. Due to the scarcity of primary vaginal cancers and the uncertainty of the primary diagnostic criteria, the clinical and pathological features, as well as the diagnostic and treatment protocols of primary vaginal 
cancer remain unclear. The aim of the study was to evaluate the clinical and pathological features, as well as treatment and prognosis of primary vaginal cancer, and to compare them with the literature.

\section{MATERIAL AND METHODS}

Sixteen patients with primary vaginal carcinoma, admitted to the Department of Gynecologic Oncology, Izmir Tepecik Training and Research Hospital between January 1983 and December 2012, were evaluated retrospectively. Patients were evaluated for age, gravidity, parity, tumor markers, pathology results, operation types, whether or not they received postoperative adjuvant therapy, prognosis, and death rate. FIGO staging was performed. The SPSS (Statistical Package for Social Sciences) program was used for statistical analyses. All data are presented in tables. OS and pelvic failure-free survival (PFFS) were estimated using the Kaplan-Meier method with two-sided log-rank test. Multivariate analyses were performed using the Cox proportional hazard model. The $p$-value of $<0.05$ was considered as statistically significant for all tests.

\section{RESULTS}

The histological distribution of the 16 patients with primary vaginal carcinoma was as follows: 9 patients (56.3\%) with SCC, 5 (31.3\%) with rhabdomyosarcoma, and $2(12.5 \%)$ with adenocarcinoma. Mean patient age was $52.87 \pm 7.83$ at diagnosis. The age distribution was between 18 and 89 years. The most common complaints included vaginal bleeding and vaginal discharge. Mean menstruation time was 35 years. Parity distribution was between 1 and 6 (mean $3.27 \pm 1.61$ ) in the SCC group, and between 0 and 8 (mean $3.33 \pm 1.95$ ) in the group with other histological types. Also, three infertile patients were detected in the group with other histological types. Coexisting diabetes and hypertension were observed in 2 (22.2\%) patients with SCC, whereas 1 (14.3\%) patient with hypertension and 1 (14.3\%) with diabetes were found in the group with other histological types. Two (22.2\%) patients in the SCC group received the diagnosis of cervical cancer and received treatment, whereas in the group with other histological types 1 patient (14.3\%) was diagnosed with cervical cancer and received treatment. Demographic characteristics of the patients are presented in Table 1.

All patients underwent surgical treatment. Radical hysterectomy/vaginectomy surgeries were performed in all 9 patients (100\%) from the SCC group. Two (28.6\%) patients had a partial vaginectomy, and 1 patient underwent pelvic exenteration in the group with other histological types. The rate of patients without metastasis was $77.8 \%$ in the SCC group but the rate of inguinal lymph node metastasis was
Table 1. Demographic characteristics of the patient

\begin{tabular}{|c|c|c|c|c|}
\hline & \multicolumn{2}{|c|}{ scc } & \multicolumn{2}{|c|}{ Non-SCC } \\
\hline & No & $\%$ & No & $\%$ \\
\hline \multicolumn{5}{|l|}{ Age (years) } \\
\hline$\leq 40$ & 1 & 11.1 & 3 & 42.9 \\
\hline $41-50$ & - & - & 1 & 14.3 \\
\hline $51-60$ & 3 & 33.3 & - & - \\
\hline $61-70$ & - & - & 2 & 28.6 \\
\hline$\geq 71$ & 5 & 55.6 & 1 & 14.3 \\
\hline \multicolumn{5}{|l|}{ Parity } \\
\hline Nulliparity & - & - & 3 & 42.9 \\
\hline Multiparity & 9 & 100 & 4 & 57.1 \\
\hline \multicolumn{5}{|l|}{ Complaint } \\
\hline Vaginal discharge & 3 & 33.3 & 1 & 14.3 \\
\hline PMB & 1 & 11.1 & 1 & 14.3 \\
\hline V. bleeding & 3 & 33.3 & 2 & 28.6 \\
\hline Mass fullness & - & - & 2 & 28.6 \\
\hline Prurigo & 2 & 22.2 & - & - \\
\hline Pain & - & - & 1 & 14.3 \\
\hline Diabetes & - & - & 1 & 14.3 \\
\hline Hypertension & - & - & 1 & 14.3 \\
\hline $\mathrm{DM}+\mathrm{HT}$ & 2 & 22.2 & - & - \\
\hline \multicolumn{5}{|l|}{ Other malignites } \\
\hline Servix ca & 2 & 22.2 & 1 & 14.3 \\
\hline Others & - & - & 1 & 14.3 \\
\hline \multicolumn{5}{|l|}{ Risk factors } \\
\hline No & 3 & 33.3 & 5 & 71.4 \\
\hline HPV & 1 & 11.1 & - & - \\
\hline Cigarette & 2 & 22.2 & 1 & 14.3 \\
\hline HPV + cigarette & 2 & 22.2 & - & - \\
\hline HPV + cigarette + alcohol & 1 & 11.1 & - & - \\
\hline V. irritation & - & - & 1 & 14.3 \\
\hline \multicolumn{5}{|l|}{ Hysterectomy history } \\
\hline$(-)$ & 6 & 66.7 & 6 & 85.7 \\
\hline$(+)$ & 3 & 33.3 & 1 & \\
\hline
\end{tabular}

found to be $2 \%$. In the group with other histological types, the rate of patients without metastasis was $71.4 \%$, pelvic lymph node metastasis rate was $14.3 \%$, and the inguinal, pelvic, and para-aortic lymph node metastasis rate was $14.3 \%$. When postoperative pathology was examined, the superiority of SCC histology was clearly visible. As far as the operation grade was concerned, grade 3 was observed in $55.6 \%$ and $57.1 \%$ of the subjects from the SCC group and the other histological types group, respectively. Patients in both groups had more advanced grade on histopathology exam. As for location, it was found that the lesions 
Table 2. Surgical stage and morphological characteristics of the patients

\begin{tabular}{|c|c|c|c|c|}
\hline \multicolumn{2}{|c|}{ SCC } & \multicolumn{2}{c|}{ Non-SCC } \\
\hline No & $\%$ & No & $\%$ \\
\hline
\end{tabular}

\begin{tabular}{|l|c|c|c|c|}
\hline Histological type & \multicolumn{3}{l|}{} \\
\hline SCC & 9 & 56.3 & - & - \\
\hline Rabdomyosarkoma & - & - & 5 & 31.3 \\
\hline Adenocarsinoma & - & - & 2 & 12.5 \\
\hline
\end{tabular}

\section{Figo stage}

\begin{tabular}{|l|c|c|c|c|}
\hline I & 1 & 11.1 & - & - \\
\hline I & 6 & 66.7 & 2 & 28.6 \\
\hline II & 2 & 22.2 & 1 & 14.3 \\
\hline III & - & - & - & - \\
\hline IV & - & - & 4 & 57.1 \\
\hline
\end{tabular}

\begin{tabular}{|c|l|l|l|l|}
\hline N stage & \\
\hline N0 & 7 & 77.8 & 5 & 71.4 \\
\hline N1 & 2 & 22.1 & 2 & 28.6 \\
\hline
\end{tabular}

\section{M stage}

Mo

9

M1

100

$3 \quad 42.9$

Tumour size

\begin{tabular}{|c|c|c|c|c|}
\hline $1-2 \mathrm{~cm}$ & 2 & 22.2 & - & - \\
\hline $3-4 \mathrm{~cm}$ & 4 & 44.4 & - & - \\
\hline$>5 \mathrm{~cm}$ & 3 & 33.3 & 7 & 100 \\
\hline
\end{tabular}

\section{Localization}

\begin{tabular}{|l|l|l|l|l|l|}
\hline Vagen upper $1 / 3$ & 7 & 77.8 & 6 & 85.7 \\
\hline
\end{tabular}

\begin{tabular}{|l|l|l|l|l|}
\hline Vagen middle $1 / 3$ & 1 & 11.1 & 1 & 14.3 \\
\hline
\end{tabular}

\begin{tabular}{|l|l|l|l|l|}
\hline Vagen lower $1 / 3$ & 1 & 11.1 & - & - \\
\hline
\end{tabular}

\begin{tabular}{|l|l|l|l|l|}
\hline Vagen ant. & 1 & 11.1 & 2 & 28.6 \\
\hline
\end{tabular}

\begin{tabular}{|l|l|l|l|l|}
\hline Vagen post. & 7 & 77.8 & 4 & 57.1 \\
\hline
\end{tabular}

\begin{tabular}{|l|l|l|l|l|}
\hline Vagen lat. & 1 & 11.1 & 1 & 14.3 \\
\hline
\end{tabular}

\begin{tabular}{|l|l|l|l|c|}
\hline Surgery & 9 & 100 & 7 & 100 \\
\hline Chemotherapy & 1 & 11.1 & - & - \\
\hline Radiotherapy & 4 & 44.4 & 1 & 14.3 \\
\hline KT + RT & 3 & 33.3 & 3 & 42.9 \\
\hline
\end{tabular}

Recurrence

\begin{tabular}{|l|l|l|l|l|}
\hline No & 4 & 44.4 & 2 & 28.6 \\
\hline Stumph & 2 & 22.2 & 2 & 28.6 \\
\hline Vagina & 2 & 22.2 & 2 & 28.6 \\
\hline Pelvic & 1 & 11.1 & 1 & 14.3 \\
\hline
\end{tabular}

Survival (months)

\begin{tabular}{|l|l|l|}
\hline OS & $27.78 \pm 17.04$ & $56.43 \pm 37.83$ \\
\hline DFS & $18.44 \pm 16.49$ & $35.14 \pm 30.98$ \\
\hline
\end{tabular}

originated in the upper third of the posterior vaginal wall. When assessing lymphovascular invasion, $44.4 \%$ and $57.1 \%$ cases were found in the SCC group and the other histological types group, respectively. We found that $66.7 \%$ of the patients in the SCC group were stage I, and $57.1 \%$ of the group with other histological types were stage IV. It was also observed that $44.4 \%$ of the patients in the SCC group underwent only radiotherapy, $11.1 \%$ only chemotherapy (12 cycles of carboplatin, 3 cycles of 5-FU), and 33.3\% chemoradiation as the adjuvant therapy. Chemoradiation and radiotherapy were applied to $42.9 \%$ and $14.3 \%$ of the patients in the other histological types group. Recurrence was detected in 5 (55.6\%) patients from the SCC group and $5(71.5 \%)$ patients from the other histological types group. Distant metastases in the SCC group were not observed, but 4 patients in the other histological types group showed metastasis to the bladder and the rectum. Surgical stage and morphological characteristics of the study population are presented in Table 2 .

\section{DISCUSSION}

Primary vaginal cancer is a rare malignant disease, constituting $1-2 \%$ of genital tumors in women [2]. SCC of the vagina is the most common histological type, and its frequency is estimated at $85 \%$ [12-14]. Histological features of the patients enrolled in our study were consistent with the literature. Primary vaginal cancer is often observed in women over the age of 60 and peaks in the range of 70-80 years. Only $30 \%$ of the affected patients are diagnosed before the age of 60 [5]. In our study, mean patient age was consistent with the literature, but the percentage of patients $\geq 71$ years of age was $14.3 \%$ in the SCC group and $55.6 \%$ in the other histological types group. Also, $42.9 \%$ of the patients in the other histological types group and $11.1 \%$ in the SCC group were $<40$ years of age. While advanced patient age correlates with recurrence, it is inversely related with disease-free survival (DFS) and overall survival (OS). In our study, patient age and DFS and OS were not significantly correlated.

The etiology of vaginal carcinoma is the same as cervical cancer. Multiple sexual partners, smoking, alcohol use, sexual intercourse at an early age, and poor socioeconomic status are considered to be risk factors for vaginal cancer [5-7]. SCC of the vagina was found to be more related with these parameters as compared to the other histological types group, which is consistent with the literature.

Numerous studies have examined the relationship between parity and vaginal carcinoma, but no significant association was found $[5-7,11]$. Parity was higher in the SCC group than the other histological types group, but the difference was not statistically significant.

Although the role of metabolic diseases such as obesity, diabetes, and hypertension in the pathogenesis of endometrial cancer has been confirmed, the same relationship is unclear for vaginal cancer. Previous hysterectomy, endome- 
triosis, chronic irritation, and cervical or vaginal radiotherapy are also among the risk factors. Three patients (33.3\%) in the SCC group and 1 patient (14.3\%) in the other histological types group underwent hysterectomy for benign reasons.

The stage is the most important prognostic factor in vaginal cancer. Epidemiology Survey Results (SEER) data give important information about the prognostic factors of primary vaginal cancer. Age, lymph node involvement, and tumor size are considered poor prognostic factors at diagnosis. On the other hand, the histological type of vaginal cancer has been reported as the most important factor but the significance of the grade remains to be fully elucidated. In our study, grade 3 differentiation rate was $55.6 \%$ and $57.1 \%$ in the SCC group and in the other histological types group, respectively. The majority of patients in both groups had high-grade lesions. There was no statistically significant difference between stage and patient age in either group $(p>0.05)$.

According to the SEER data, if the tumor diameter is $<4 \mathrm{~cm}$, the 5 -year survival is $84 \%$. If tumor diameter is $>4 \mathrm{~cm}$, the 5 -year survival is $65 \%$. In our study, there was no statistically significant relationship between survival and tumor size. Squamous cell histology is seen in more early-stage tumors, whereas other histological types are often detected in the advanced stages (SEER). Stage 0-I was detected in $77.8 \%$ of the subjects in the SCC group, while $28.6 \%$ of the patients in the other histological types group had stage II tumors and $57.1 \%$ had stage IV tumors. This ratio is consistent with the general literature and SEER data.

Lymphovascular invasion (LVSI), regardless of histological grade or stage, is a powerful indicator of tumor recurrence and death. Vascular invasion suggests the presence of lymph node metastasis. In our study, DFS was significantly lower in the SCC group as compared to other histological types group if LVSI was present $(p<0.05)$.

There is no consensus in the literature regarding the treatment of primary vaginal cancer. Treatment should address individual characteristics of the patient, i.e. age, general medical condition, stage, and tumor location. Although radiotherapy is recommended as the standard treatment for all stages in many centers, surgical treatment is a better solution for early-stage disease in terms of survival [15-17]. Radical hysterectomy, vaginectomy, and pelvic lymph node dissection are the current approaches to treating vaginal cancer $[12,13,16,17]$. Vaginal cancer management has not been standardized because prospective studies on the possible treatment of vaginal cancer have not been conducted. There are no uniform treatment options, and a highly individualized treatment is necessary. The generally accepted standards of radical surgical modality include:

- partial vaginectomy with adequate surgical margins + pelvic lymphadenectomy \pm hysterectomy for mass located in the superior part of the vagina,
- radical vaginectomy with vulvectomy and inguinal lymphadenectomy for vaginal lesions located in the inferior part of the vagina,

- radical vaginectomy + pelvic lymphadenectomy + elective inguinal lymphadenectomy (only palpable inguinal nodes) are recommended for lesions located in the middle part of the vagina $[12,16,18]$.

All patients in our study underwent radical surgery: $44.4 \%$ in the SCC group underwent only radiotherapy, $11.1 \%$ only chemotherapy (12 cycles of carboplatin, 3 cycles of 5-FU), and 33.3\% chemoradiation as the adjuvant therapy. Chemoradiation was applied to $42.9 \%$ and radiotherapy was applied to $14.3 \%$ of the patients in the other histological types group.

\section{CONCLUSIONS}

The stage of the disease constitutes the primary factor influencing DFS and OS. We could not reach statistically significant conclusions whether age, parity, or age at menarche and menopause are risk factors for vaginal cancer. Thus, medical history of the patients and our findings were analyzed and compared with the literature. The evaluated parameters were consistent with the literature. We concluded that there is a need for more extensive and multicenter studies to achieve valid results because of the limited number of cases with vaginal cancer.

\section{REFERENCES}

1. Graham JB, Meigs JV. Earlier detection of recurrent cancer of the uterine cervix by vaginal smear. Am J Obstet Gynecol. 1952, 64 (4), 908-914.

2. Brunner AH, Grimm C, Polterauer S, [et al.]. The prognostic role of human papillomavirus in patients with vaginal cancer. Int J Gynecol Cancer. 2011, 21 (5), 923-929.

3. Ragni MV, Tobon H. Primary malignant melanoma of the vagina and vulva. Obstet Gynecol. 1974, 43, 658.

4. Hilborne LH, Fu YS. Intraepithelial, invasive and metastatic neoplasms of the vagina. In: Wilkinson EJ, ed. Pathology of the vulva and vagina. New York, Churchill Livingstone 1987, 184.

5. DiSaia PJ, Creasman WT. Clinical gynecologic oncology, $6^{\text {th }}$ ed. Chapters 2 and 9. St Louis, Mosby 2002.

6. Sinha B, Stehman F, Schilder J, [et al.]. Indiana University experience in the management of vaginal cancer. Int J Gynecol Cancer. 2009, 19 (4), 686-693.

7. Malmström $\mathrm{H}$, Engquist $\mathrm{M}$. Primary invasive cancer of the vagina. Int J Gynecol Cancer. 1997, 7 (3), 205-212.

8. Jones HW. Malignancies of the vulva and vagina. In: Jones HW, Wentz AC, Burnett LS, eds. Novak's textbook of gynecology. Baltimore, Williams and Wilkins 1988, 599-622.

9. Nomura $\mathrm{H}$, Matoda $\mathrm{M}$, Okamoto $\mathrm{S}$, [et al.]. Clinical characteristics of non-squamous cell carcinoma of the vagina. Int J Gynecol Cancer. 2015, 25 (2), 320-324.

10. Pecorelli S, Benedet JL, Creasman WT, [et al.]. FIGO staging of gynecologic cancer. 1994-1997 FIGO Committee on Gynecologic Oncology. International Federation of Gynecology and Obstetrics. Int J Gynaecol Obstet. 1999, 65, 243-249.

11. Rock JA, Jones HW, eds. TeLinde's operative gynecology. $10^{\text {th }}$ ed. Chapter 2. 2013,67 .

12. Stock RG, Chen AS, Seski J. A 30-year experience in the management of primary carcinoma of the vagina: analysis of prognostic factors and treatment modalities. Gynecol Oncol. 1995, 56, 45-52.

13. Otton GR, Nicklin JL, Dickie GJ, [et al.]. Early-stage vaginal carcinoma: an analysis of 70 patients. Int J Gynecol Cancer. 2004, 14, 304-310. 
14. Hellman K, Silfversward C, Nilsson B, [et al.]. Primary carcinoma of the vagina: factors influencing the age at diagnosis. Int $J$ Gynecol Cancer. 2004, 14, 491-501.

15. Otton G, Nicklin J, Dickie G, [et al.]. Early-stage vaginal carcinoma — an analysis of 70 patients. Int J Gynecol Cancer. 2004, 14 (2), 304-310.

16. Herwig $\mathrm{K}$, Ulrike $\mathrm{M}$, Thomas $\mathrm{H}$, [et al.]. Radiotherapy alone for invasive vaginal cancer: outcome with intracavitary high dose rate brachytherapy versus conventional low dose rate brachytherapy. Acta Obstet Gynecol Scand. 2001, 80, 355-360.

17. Davis KP, Stanhope CR, Garton GR, [et al.]. Invasive vaginal carcinoma: analysis of early-stage disease. Gynecol Oncol. 1991, 42, 131-136.

18. Rubin SC, Young J, Mikuta JJ. Squamous carcinoma of the vagina: treatment, complications, and long-term follow-up. Gynecol Oncol. $1985,20,346-353$. 